DOI: $10.35643 /$ Info.25.1.7

Artículo Original

\title{
Fotografía Film Still: el Cine Universitario visto a través de su acervo fotográfico
}

\section{Film Still Photography: Cine Universitario seen through its photographic collection}

\author{
María Eugenia Arrejuría ${ }^{1}$ \\ María Eugenia Bonino \\ Alessandra Cicero ${ }^{3}$ \\ Daniella Rampa ${ }^{4}$ \\ Daniela Rodríguez ${ }^{5}$ \\ Niza Trujillo ${ }^{6}$
}

\begin{abstract}
${ }^{1}$ Estudiante avanzada de la Licenciatura en Bibliotecología del Instituto de Información (Facultad de Información y Comunicación, Universidad de la República, Uruguay). ggmaruarhe@hotmail.com. ORCID: 0000-0002-0720-0238.

${ }^{2}$ Estudiante avanzada de la Licenciatura en Bibliotecología del Instituto de Información (Facultad de Información y Comunicación, Universidad de la República, Uruguay). eubonny@gmail.com. ORCID: 0000-0002-1314-9897.
\end{abstract}

${ }^{3}$ Estudiante avanzada de la Licenciatura en Bibliotecología del Instituto de Información (Facultad de Información y Comunicación, Universidad de la República, Uruguay). alesscicero@gmail.com. ORCID: 0000-0002-9567-1178.

${ }^{4}$ Estudiante avanzada de la Licenciatura en Bibliotecología del Instituto de Información (Facultad de Información y Comunicación, Universidad de la República, Uruguay). danirampa1970@gmail.com. ORCID: 0000-0001-8778-5493.

${ }^{5}$ Estudiante avanzada de la Licenciatura en Bibliotecología del Instituto de Información (Facultad de Información y Comunicación, Universidad de la República, Uruguay). dani.rodriguez.uy@gmail.com. ORCID: 0000-0002-2362-8525.

${ }^{6}$ Estudiante avanzada de la Licenciatura en Bibliotecología del Instituto de Información (Facultad de Información y Comunicación, Universidad de la República, Uruguay). trujilloniza@gmail.com. ORCID: 0000-0003-1472-3210.

\section{Resumen}

La fotografía film still tuvo un papel clave entre las décadas de 1920 y 1950 como una extensión visual de la industria cinematográfica durante sus inicios. Sin embargo, la literatura sobre este material fotográfico es injustamente escasa. Esta investigación se llevó a cabo en el Cine Universitario entre abril y octubre de 
2019 y ofrece una perspectiva sobre la fotografía film still, su historia y características como documento visual.

Se realizó una revisión bibliográfica para mejor comprender y conceptualizar este género fotográfico y luego se comparó con una muestra de 257 film stills de películas de los años 50 que forman parte del acervo de film stills del Cine Universitario. Los resultados se presentan en profundidad. Para celebrar el septuagésimo aniversario del Cine Universitario como uno de los últimos cineclubes en Uruguay, incluimos entrevistas con tres de sus miembros clave como representantes de la historia oral y la cultura cinematográfica de la institución.

Se extiende la discusión sobre el estudio de la imagen en el dominio de la Ciencia de la Información y Bibliotecología. Compartimos hallazgos que sirven a conversaciones actuales sobre nuevas perspectivas teórico-metodológicas disponibles para innovar y mejorar el panorama de nuestra disciplina en la era digital de la información.

Palabras clave: FOTO FIJA; DOCUMENTACIÓN AUDIOVISUAL; ANÁLISIS DOCUMENTAL; CINE UNIVERSITARIO; CULTURA CINEMATOGRÁFICA.

\section{Abstract}

Film Still photography had a key role between the 1920s and 1950s as a visual extension of the film industry's early beginnings, yet literature on this photographic material is unfairly scarce. This research project took place at Cine Universitario between April and October 2019 and provides an in-depth view on film still photography, as well as on its history and characteristics as a visual document.

A bibliographic research was conducted to better understand and conceptualize this photographic genre and was then compared to a sample of 257 film stills photographs from 1950's motion pictures that make up part of Cine Universitario's film still photographic collection. Results are thoroughly presented. Celebrating Cine Universitario's $70^{\text {th }}$ anniversary as one of the last standing film societies in Uruguay we included interviews with three of its key 
members as representatives of Cine Universitario's oral history and cinema culture.

Further discussion on image studies within the Library and Information Science domain is provided. We share purposeful findings to serve the conversations taken place today on new theoretical and methodological perspectives available for innovating and improving our discipline's landscape within Information's digital era.

\section{Keywords: FILM STILLS; AUDIOVISUAL DOCUMENTATION; DOCUMENTAL ANALYSIS; CINE UNIVERSITARIO; CINEMA CULTURE.}

Fecha de recibido: $30 / 03 / 2020$

Fecha de aceptado: 27/04/2020

\section{Introducción}

La presente investigación fue desarrollada por estudiantes de la Licenciatura en Bibliotecología de la Facultad de Información y Comunicación (FIC - Udelar) con el interés de profundizar en el estudio del género fotográfico film still como documento visual y en las herramientas posibles de ser aplicadas a su tratamiento documental en la era digital de la información. La misma es producto de dos instancias previas de trabajo, realizadas en los marcos del Espacio de Formación Integral (EFI) $^{1}$ y de Extensión Universitaria ${ }^{2}$ de la FIC, en los años 2017 y 2018 respectivamente. En estas, conocimos el acervo fotográfico del Cine Universitario (CU), identificando el género 'film still' o 'foto fija' que lo compone como tal.

Estos stills suponen un volumen estimado de 20.000 ejemplares aproximadamente. Desde el 2017 venimos desarrollando prácticas documentales a fin de diseñar un tratamiento documental sostenido en el tiempo que logre su articulación con los lineamientos actuales del CU como casa cultural de cineclubismo.

Los insumos reunidos en las experiencias previas permitieron realizar el presente proyecto de investigación tras ser aprobado y financiado por la Comisión Sectorial de Investigación Científica (CSIC) en el marco del Programa de Apoyo a la Investigación Estudiantil (PAIE) ${ }^{3}$; el mismo se tituló "Film Stills: la fotografía como memoria visual del Cine Universitario" y se ejecutó durante los 
meses abril-octubre del 2019.

Habiendo identificado al film still como el género fotográfico que compone al acervo, nos interesó profundizar en su estudio para una mejor comprensión de este material y diseñar la proyección de su tratamiento documental. La naturaleza visual del film still y su rol histórico en el fortalecimiento de la construcción y desarrollo de la industria cinematográfica a principios del siglo XX sugirieron el punto de partida de nuestro estudio.

Este implicó enfocar las herramientas teórico-metodológicas de nuestra disciplina, Ciencia de la Información y Bibliotecología en dos dimensiones: la primera remite al carácter interno del acervo de stills del CU, actualmente inaccesible al público y que demanda un estudio propio al campo de la Documentación Audiovisual, particularmente centrado en el análisis y tratamiento de la imagen como documento.

Por su parte, el carácter externo, comprende al contexto en el cual encontramos dicho acervo fotográfico. Este último hace parte de la colección audiovisual de la Biblioteca y Archivo del CU, la cual tiene un potencial reconstructivo sobre la historia institucional y comunitaria del CU, inaugurado en 1949 con el fin de celebrar y construir la cultura cinematográfica del Uruguay. En este sentido, el acervo estudiado supone un puente significativo para traer al presente el registro del pensar y quehacer fílmico de la institución con el fin de revitalizar y visibilizar la memoria cinematográfica de su comunidad.

Desde esta perspectiva, nuestra investigación se propuso un trabajo proyectable a futuro que consiga conjugar ambas dimensiones en un programa de gestión y producción cultural que acompañe al desarrollo y realización de los objetivos institucionales del CU.

\section{Desarrollo}

\subsection{El film Still o la imagen como documento}

Alcanzando su auge entre los años 1920 y 1950 en los Estados Unidos, el film still se definió como la fotografía diariamente tomada de una escena durante o finalizada la filmación de la misma a fin de contar con un registro visual del proceso creativo de la película para su eventual marketing y comercialización (Gomery, 2004). Muchas veces, los film stills capturaban escenas no incluidas en 
el corte final de la película, o dejaban ver momentos tras bambalinas de la producción, enriqueciendo así el registro visual de la misma (véase ilustración 1).

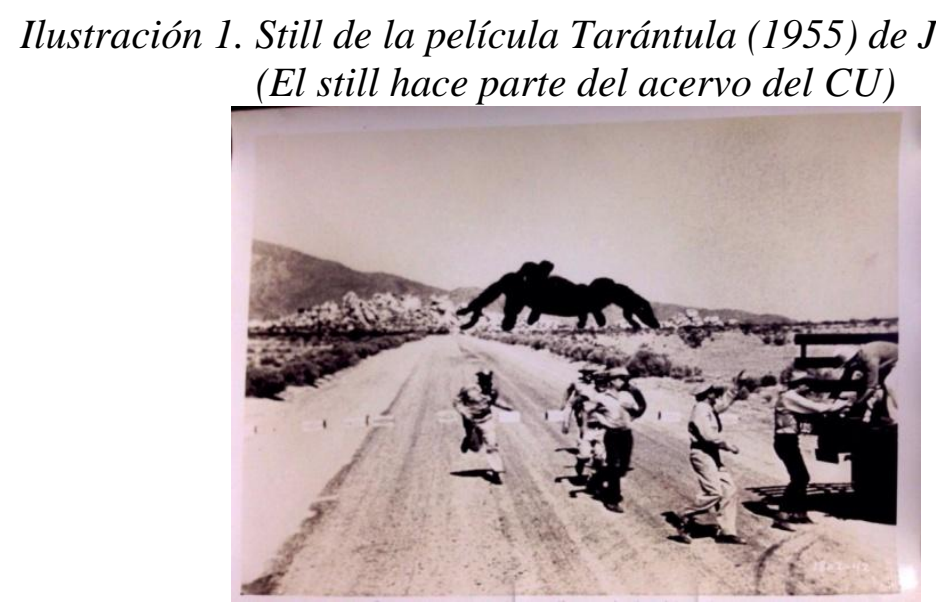

Fuente: Universal Pictures Company Inc.

En ese entonces, la baja calidad ofrecida por el fotograma o 'film grab' y la necesidad de economizar los tiempos de revelado y difusión del material como referencia interna en los departamentos de filmación (maquillaje, vestimenta, iluminación, entre otros), convirtieron al film still en un instrumento clave para la historia visual del cine que injustamente ha sido poco mencionado en la literatura. El still era tomado en el set por el stillman, fotógrafo - referido como una figura silenciosa y poco bienvenida en el set - que era contratado por el departamento de publicidad de las grandes productoras cinematográficas de la época; estas podían llegar a demandar una tirada de 300 stills diarios. Luego pasaban al laboratorio de revelado y edición donde se 'retocaban' las fotografías y se reproducían los negativos lo más económicamente posible para su distribución masiva e inmediata.

Al comprar las películas, los cines y teatros de ese entonces adquirían con estas un kit de prensa (press-kit/ press-book) conformado por film stills, diapositivas, pósteres y resúmenes para exhibir en sus lobbies y halls durante el período en el que se proyectasen las mismas (Blanchard, 1930 ; MacKenzie, 1934). Asimismo, prensa, diarios y revistas de la época también hacían uso de stills como complemento visual para sus reseñas y comentarios. Actualmente son utilizados como fuentes de información de apoyo por ejemplo, para los estudios fílmicos (Film Studies) en el ámbito académico.

Con el paso del tiempo, mas el perfeccionamiento y digitalización del medio 
cinematográfico, los modos de ver y consumir cine cambiaron dejando al film still en un rol secundario y aún más desapercibido en la literatura del que supo tener en sus inicios. Sin embargo, su funcionalidad en cuanto al registro de las producciones cinematográficas pone en manifiesto el carácter documental e informativo que supone la imagen como objeto de estudio.

En consecuencia, para la Ciencia de la Información y Bibliotecología el problema de la imagen en el siglo XXI supone una necesaria revisión histórica y paradigmática de las herramientas teórico-metodológicas que pueden ofrecerse a la comprensión de las transformaciones que los mecanismos tecnológicos y comunicativos han realizado sobre el tejido social y su relacionamiento con la información. Así, la imagen como objeto de información se ha convertido tanto en un fenómeno masivo como también en un documento histórico capaz de registrar y construir una narrativa instantánea. (Burke, 2005 ; Meza Castro, 2018). Para nuestra disciplina, la fotografía encarna esta descripción.

Desde la perspectiva de la Documentación Audiovisual, encontramos en la imagen fotográfica un tipo de documento con particularidades inherentes a su naturaleza visual, a los distintos - y cambiantes - formatos de su presentación, a las dimensiones espacio-temporales que rigen en la misma y su poder constructivo de memoria social (Caridad, Hernández Pérez, Rodríguez y Pérez, 2011 ; Freund, 2017 ; Solórzano-Ariza, Toro-Tamayo Vallejo-Echavarría, 2017).

Nuestro objeto de estudio constituye una memoria por tanto, cinematográfica en tanto registra el proceso de la producción fílmica como tal por un lado, y por otro, una memoria institucional ya que es representativa de una relación específica entre sociedad e información en torno a la cultura cinematográfica de la comunidad cineclubista del CU. En Uruguay, el CU y su comunidad representan un espíritu comprometido históricamente con el desarrollo y ejercicio de cultura cineclubista, promoviendo la crítica y reflexión sobre el lenguaje fílmico desde hace 70 años. Su acervo fotográfico hace parte de la representación visual de esa historia.

Así, este proyecto se enfoca en el estudio de la fotografía film still como documento visual traducido al análisis del acervo de stills que constituye parte de la colección audiovisual del CU para luego profundizar en cómo recuperar la memoria cinematográfica que este material puede revelar sobre la institución y su 
comunidad en el tiempo.

\subsection{Objetivos}

El principal eje que sostiene el interés y pertinencia de esta investigación nace en el deseo de conciliar la escasa literatura existente sobre el género fotográfico film still y su comparación con el acervo de film stills del CU para su conceptualización y posible tratamiento documental en el marco digital de la información. En consecuencia, este obstáculo pasó a centrar la necesidad de desarrollar el concepto y características del film still como documento, las implicaciones que este tiene en cuanto a su tratamiento y qué tipo de correspondencias teórico-prácticas podrían identificarse en el acervo de stills del CU para su futura recuperación y gestión. En este sentido, nos propusimos explorar los siguientes objetivos:

General

- Desarrollar insumos teóricos - metodológicos propios a la Ciencia de la Información y Bibliotecología que contribuyan a la reconstrucción histórica-documental del Archivo y Biblioteca del Cine Universitario.

Específicos

- Estudiar y analizar el género fotográfico film still como documento visual y sus correspondencias teórico- prácticas con el acervo de stills del CU.

- Analizar una muestra de 200 stills del acervo del CU que consolide un primer acercamiento a herramientas posibles de aplicar a su tratamiento documental.

- Identificar en la colección general del CU (libros, revistas, folletos, actas institucionales, etc.) posibles documentos y/o registros que traten sobre film stills o brinden información sobre el acervo estudiado, su relación con la institución y cuál fue su uso.

- Entrevistar actores clave de la comunidad del CU que signifiquen fuentes de información pertinentes a construir conocimiento sobre el género film still, el CU y su comunidad.

\subsection{Metodología}

La propuesta metodológica se diseñó desde el modelo de investigación - acción 
(Kemmis y McTaggart, 1988). El mismo se justificó desde la decisión del equipo de abordar el análisis del acervo de stills del CU como un objeto de estudio anexado a la historia de la institución y su comunidad. Este componente pone en manifiesto también la interpretación de nuestra disciplina en cuanto a su rol no solo social y cultural, trabajando cerca y en conjunto con diversos actores que participan en la creación de conocimiento, sino también enfocado en la construcción de bases conceptuales sólidas que enriquezcan e innoven el quehacer de nuestra profesión.

Dada la magnitud del acervo, trabajamos sobre una muestra del mismo previamente relevado en instancias anteriores de trabajo (EFI y Extensión Universitaria). Dada la escasa literatura con la que contábamos hasta entonces, el modelo investigación-acción permitió una estrategia paulatina de estudio teórico y traducción práctica a líneas de acción que garantizaran el mejoramiento del acervo y su posible articulación con los lineamientos actuales del CU. Para ello, la investigación se organizó en cinco etapas:

1. Revisión bibliográfica sobre Film Still

2. Correspondencias entre la literatura encontrada y el acervo de film stills del CU

3. Entrevistas con actores clave del CU

4. Análisis de resultados finales

5. Conclusiones y Proyección

El desarrollo realizado y hallazgos encontrados en cada una de ellas se detalla a continuación:

\section{Resultados}

\section{1. 'Film Still' y 'Foto Fija': dos perspectivas histórico-documentales}

Como mencionamos anteriormente, la literatura que existe expresamente sobre film still es escasa. Los artículos encontrados que profundizan en la temática fueron producidos durante la época de auge de este género fotográfico (1920 1950), y datando los producidos entre los años 20 - 30. Si bien el material encontrado luego de este período fue analizado, no se identificó al film still como eje central del mismo, excluyendo algunos trabajos puntuales donde sí fue enfocado como tal. 
Cabe destacar a su vez, que la revisión bibliográfica se realizó teniendo por referencia inicial al término "film still” y por tanto, significó la recuperación de literatura mayoritariamente en el idioma inglés. Esto también causó una concentración al inicio del estudio en torno a la industria cinematográfica estadounidense donde el film still y el fotógrafo stillman tuvieron un protagonismo clave durante el período 1920 - 1950. Blanchard (1930 : 12) señala así su pertinencia:

«[...] una still, vista en las revistas y diarios o exhibidas en los lobbies de los teatros y cines, es casi el único criterio por el que el público puede juzgar los méritos visuales de la producción.»

En este sentido, haber encontrado artículos publicados durante la misma época donde la fotografía film still comenzaba a desarrollarse como tal, nos provee una perspectiva socio-histórica significativa para comprender el valor que esta tenía para el público. Por un lado, interpretamos en el comentario de Blanchard una cercanía entre la fotografía film still y el público, siendo la primera un objeto anticipativo de la película propiamente dicha y de gran visibilidad (revistas, diarios, lobbies y halls de teatros y cines) (véase ilustración 2). Por otro, nos cuenta sobre el valor visual y crítico que estas fotografías dictaban sobre el público y su relacionamiento con el cine.

\section{Ilustración 2. ALEXIS. Teatre i Cinema. Rambla Catalunya. 90 (1954-2013)}

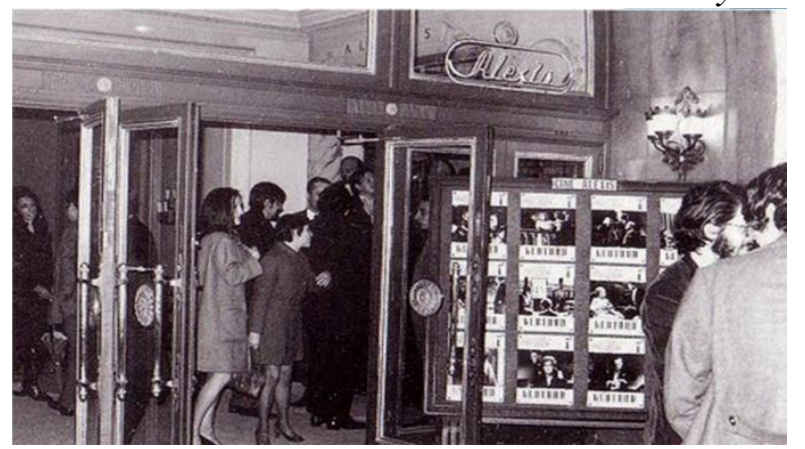

Fuente: Barcelofília

Proponemos comparar al film still en este sentido como el 'trailer' de aquella época, el ‘primer vistazo' que generaba una conexión entre el público y la película que iban a ver. A su vez, Mackenzie (1934) atribuye este resultado a la efectividad del fotógrafo stillman en cuanto a su capacidad de capturar la foto que mejor pudiese contar y promocionar el argumento de la película.

Casualmente, en Once Upon a Time in Hollywood del director Quentin Tarantino 
(2019), se puede encontrar una escena donde se muestra el uso del film still en las carteleras y vitrinas de cines y teatros (véase ilustración 3). En ella, la actriz Sharon Tate (interpretada por Margot Robbie) se encuentra en la boletería del teatro Bruin, en donde se estrena la película The Wrecking Crew (1968) que coprotagoniza junto a Dean Martin. Tate, le señala una lobby card (tipo de film still a color impreso en papel o cartulina gruesa) a la vendedora de boletería donde se ve a la actriz en una escena de la película causando así su reconocimiento y dejándola ingresar a la sala gratuitamente.

Ilustración 3. Proud: The 28-year-old Oscar-nominated actress puts on an impressive performance as the relatively bubbly Tate who goes to the movies to watch herself in 1968 Dean Martin film

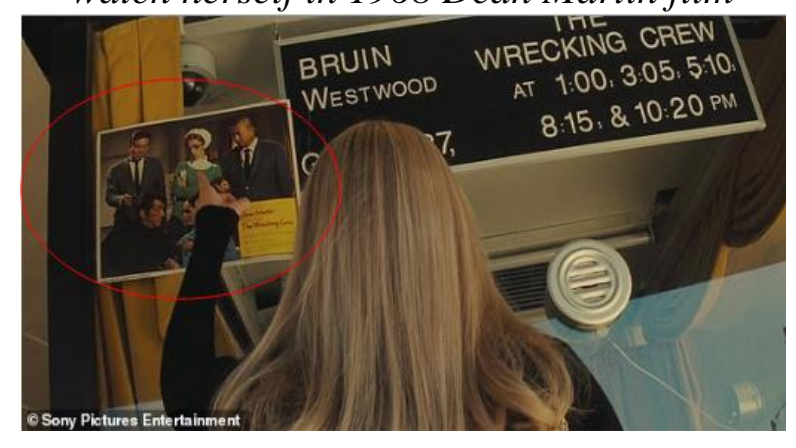

The Wrecking Crew. Fuente: Sony Picture Entertaiment

Así, el valor estético de la composición de la fotografía still también sirvió al desarrollo del sistema de estrellas hollywoodense (star system) y supuso la consecuente iconografía representada y promovida por la industria del cine. En este sentido, la calidad del still era clave en el momento de la selección de fotografías que componían el portfolio de marketing de la película en el que se apoyaban los cines y teatros para su compra y eventual exhibición, reconociendo el poder informativo y comunicativo de la imagen sobre el individuo (Jacobs, 2010).

Al mismo tiempo que el film still instrumentó la presentación publicitaria de las películas ante el público, también significó un material referencial durante el proceso creativo de las mismas. Archer y Freyer (1928) sintetizan cuatro funciones que cumplían las fotografías film still: publicitario (estadio más visible en revistas y diarios, lobbies y halls de cines y teatros); marketing (un estadio menos visible pero clave en las estrategias de venta de las películas a los teatros y cines de la época a través del portfolio de stills de las mismas); referencial 
(estadio interno durante la producción de la película para mantener coherencia y cohesión estética entre los departamentos de maquillaje, vestuario, iluminación, etc.); trick photography o 'trucos fotográficos' (comprendían el uso de stills como 'engaño' visual por ejemplo utilizados como background image mientras se anunciaban los créditos de la película).

Diferenciamos estas funciones como propias al concepto clásico de film still comprendido entre los años 1920 - 1950. Si bien nuestro estudio se centra en este período, advertimos un cambio conceptual y técnico del film still a partir de los años 60 hasta el presente, subyugado a los avances tecnológicos del medio fílmico. Estos han posibilitado -entre otras cosas - la digitalización de la imagen y nuevas formas de consumo cinematográfico por ejemplo. Por tanto, el registro y uso tradicional del film still en vitrinas y carteleras de cines y teatros es casi inexistente desde entonces (Virué Escalera, 2012).

Hasta aquí situamos al film still clásico dentro del contexto de la industria cinematográfica estadounidense. En consecuencia, nos interesó traducir la búsqueda del término film still al idioma español para observar qué tipo de resultados podrían obtenerse, encontrando así su equivalente: foto fija o fotografía fija. Este término cuenta con las mismas características definidas para film still, al cual se referencia históricamente aunque con menos precisión conceptual e incluso más carencias en cuanto a su estudio científico. No encontramos en esta instancia material suficiente para identificar una historia de foto fija como tal, como logramos con el término film still.

En este sentido, la mayor parte del material encontrado remite a entrevistas o artículos no científicos sobre fotógrafos encargados de la fotografía fija en producciones cinematográficas iberoamericanas; más sí existe consenso sobre la subestimación del stillman o 'fotofijas' y su rol poco conocido fuera de la industria del cine (García Torres, 2013).

Aún así, los resultados encontrados sobre foto fija revelan algunos aspectos interesantes al contrastarlos con el enfoque clásico del film still estadounidense. Mientras el último es conceptualizado como herramienta publicitaria - y ciertamente con un propósito de uso breve y puntual -, el enfoque sobre el término foto fija sugiere además una mirada sobre su dimensión socio-cultural.

En este sentido, encontramos en el paradigma iberoamericano un marcado interés 
sobre la construcción de la cultura visual y cinematográfica de los públicos y cómo impactan en su identidad a través de la imagen, viralizada a través de nuevas tecnologías que han cambiado su reproducción y masificación, convirtiéndose así en un fenómeno independizado de la película en sí misma. (Tuñón, 2002 ; Virué Escalera, 2012 ; Flores Huelves \& Montes Vozmediano, 2017). Este paradigma encontraría su razón en la herencia de una cultura cinematográfica tradicionalmente europea con raíces claras en el cineclubismo y cinefilia como expresiones sociales que trascienden la experiencia comercial del cine para transformarla en "una acción de cultivar el placer cinematográfico" (Broitman y Eseverri, 2018 : 166).

Al traducir esta perspectiva a nuestra investigación, encontramos un puente conceptual propicio para aplicar al acervo de film stills del CU que, desarrollado como cineclub desde sus inicios se sostiene en su carácter no solo de cine nocomercial, sino también como espacio de transformación e interacción social $a$ través del cine. Esta característica se refuerza a su vez, al contar con una colección audiovisual especializada en cinematografía, siendo su acervo de film stills una parte de su cultura cinéfila y coleccionista de distintos tipos de material cinematográfico.

Desde nuestra disciplina, esto implica trabajar hacia la recuperación del acervo, reconstruyendo cómo se fue formando como tal en el tiempo y las relaciones que este tiene con la literatura consultada. Para esta investigación nos detuvimos a identificar correspondencias teórico-metodológicas entre la literatura analizada y las características del acervo del CU.

\subsection{El acervo de film stills del Cine Universitario y su tratamiento documental}

El estudio del acervo de film stills del CU se desarrolló a partir de tres ejes conceptuales nacidos de la literatura consultada en la primera etapa de la investigación. Estos son:

A. El concepto del film still clásico comprende los años 1920 - 1950 en el marco de la industria cinematográfica estadounidense.

B. El uso del film still tuvo un propósito expresamente publicitario.

C. El género fotográfico film still se singulariza por características específicamente adaptadas a las necesidades de la industria 
cinematográfica.

Recordamos que, se estima que el acervo del CU cuenta con unos 20.000 film stills aproximadamente. Estos están guardados en sobres por orden alfabético bajo apellido de director. De este volumen inicial, hemos relevado e inventariado 2213 ejemplares (período 2017-2018), consignando datos clave para su recuperación: título de película, director, año de producción, país de producción, productora, actores en escena, entre otros. Más del $40 \%$ de estos stills relevados pertenecen a películas de los años 50 (901 stills en total). En consecuencia, la traducción metodológica de los tres ejes conceptuales se diseñó de la siguiente manera:

a. Selección aleatoria de film stills pertenecientes a películas de los años 50: Dado los tiempos y alcances estipulados para la investigación, el estudio del acervo se realizó sobre una muestra total de 257 film stills de los 901 ejemplares previamente relevados, pertenecientes a películas de la década de 1950. La elección de este período radicó principalmente en el reconocimiento de la historia del CU y la 'época dorada' que vivió su comunidad ante la coyuntura favorable de la sociedad uruguaya durante esta década y por tanto, en una creciente cultura cinematográfica que encontró terreno fértil en su propuesta institucional, acompañada por el cineclubismo emergente en la región (Costa, J. \& Scavino, C., 2009), lo cual nos brindó un marco referencial claro donde contextualizar al acervo y su posible uso durante esta época.

Por su parte, para la industria cinematográfica estadounidense, la década del 50s supuso el fin de una era donde convergieron "el divorcio de los principales estudios de sus teatros y la pérdida de una gran parte de la audiencia cinematográfica" (Boddy, 1985: 23), que cambió sus hábitos de consumo ante la creciente popularidad de la televisión. Así, nos situamos en un contexto sociohistórico que demanda tener presente el desarrollo del cine dentro de la agenda política internacional estadounidense, la cual puso en marcha una industria cultural donde la imagen se convirtió en un vehículo de difusión masiva del 'estilo de vida americano' para todo el mundo, agudizando así el ingreso por ejemplo de más producciones cinematográficas, (y por tanto, material anexo como film stills) en la región.

La llegada de este tipo de material y su uso - al menos en el CU - no quedó limitado a fines publicitarios, sino que se transformó en objeto de colección 
(contrario a su propósito de uso y descarte tradicional). Esto nos permite hoy estudiar un acervo que nos cuenta dos narrativas entrelazadas: el tipo de cine que se importaba y consumía en Uruguay y el posible relacionamiento que su audiencia tuvo con este en el caso del CU.

b. Análisis de la muestra: el uso del film still en el Cine Universitario: En consecuencia, la muestra analizada revela correspondencias con la literatura consultada (Gomery, 2004 ; Jacobs 2010). Los film stills analizados presentan marcas de uso, de las cuales diferenciamos dos tipos: pliegues y agujeros de alfileres en los márgenes, y anotaciones y sellos al dorso de la fotografía.

El primer tipo de marcas que llamaremos tipo A, comparten similitudes con los usos descritos en cuanto a su exhibición en carteleras y vitrinas de cines y teatros. La presencia de este tipo de marcas en la muestra es frecuente, lo cual nos permite estimar que las fotografías tuvieron un nivel alto de exhibición. Las anotaciones(véase punto c) y sellos al dorso (marcas tipo B), suponen información un tanto más rica en cuanto nos cuentan cómo se fue conformando el acervo como tal (es decir, cómo llegaron a incorporarse al acervo actual). Las marcas tipo B fueron sistematizadas como datos clave para la contextualización histórica del acervo, estas son:

- Sellos de diarios, publicaciones periódicas: La Mañana y El País.

- Sellos de instituciones cineclubistas o relacionadas a la esfera cinematográfica: Cine Universitario, Cine Club del Uruguay, Films Mundiales del Uruguay.

- Productoras y entidades cinematográficas: 20th Century Fox, Paramount Pictures, Metro Goldwyn Mayer, Continental Films, Astral Films, International Films.

- Entidades nacionales: Consejo del Niño.

Los sellos encontrados corroboran la utilización del film still en medios y prensa para publicitar las películas como es mencionado en la literatura. A su vez, nos encontramos con sellos de instituciones cineclublistas o relacionadas al cine; estos dos tipos de sellos revelan las fuentes de procedencia de la muestra.

En cuanto al país de origen de las productoras y entidades cinematográficas, vemos una marcada presencia de producciones estadounidenses, seguida de las francesas. Esto nos parece interesante tratarlo desde un marco socio-económico, 
ya que la fotografía film still, si bien se desarrolló en iberoamérica y en países europeos, no contó con la misma infraestructura y recursos que caracterizó a la industria estadounidense. Esta observación se vuelve muy clara cuando nos detenemos a analizar dentro de la muestra a la representación de los film stills analizados por país de origen (véase gráfica 1).

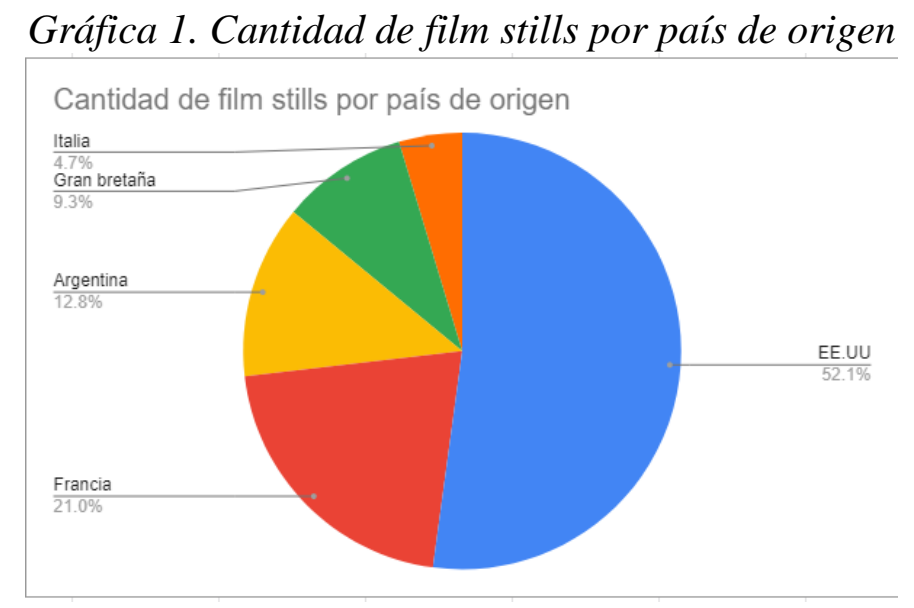

Elaboración propia.

Asimismo, la presencia de producciones argentinas representa el único país latinoamericano en la muestra $(12,8 \%)$, por debajo de Francia $(21 \%)$ y Estados Unidos $(52,1 \%)$. Desde esta perspectiva no solo podemos observar posibles tendencias de consumo de la época, sino también una economía internacional igual de significativa para comprender su contexto.

Por último, destacamos el hallazgo de sellos del Consejo del Niño, lo cual abrió una nueva dimensión de la investigación (en relación a políticas estatales de control y censura en el cine en Uruguay) aún no explorada; en esta instancia solo haremos alusión a su hallazgo, ya que su estudio excede los propósitos de este trabajo.

c. Definición de las características fotográficas del género film still Una vez reunidas las características históricas que la muestra mantiene en relación específica con el CU, nos detuvimos a analizar las características documentales que corroboran su categorización dentro del género clásico del film still. Entre ellas destacamos: dimensiones formales: $20 \times 25 \mathrm{cms}$; fotografía en blanco y negro ; impresión en papel fotográfico; presencia de pie de foto.

La pluralidad de usos y presentaciones del film still puso en manifiesto la necesidad de homogeneizar la descripción documental de este tipo de fotografías 
para luego formalizar un modelo de tratamiento documental adecuado al acervo del CU. Teniendo presente la literatura consultada (Archer \& Freyer, 1928 ; Gomery, 2004), la muestra coincide con las dimensiones clásicas de 20 × 25 centímetros (u 8 x 10 pulgadas); la mayoría de los film stills analizados son fotografías en blanco y negro como era de estilar (aunque encontramos lobby cards por ejemplo, también reconocidas en la literatura, impresas en color y en un material más fuerte como el cartón para ser exhibidas a distancias más cercanas del público); la calidad de impresión del papel fotográfico de la muestra es muy buena, lo cual facilita futuros trabajos de conservación y preservación documental.

También corroboramos la presencia del pie de foto en la mayoría de la muestra. Este contiene datos sobre la producción de la película a la que pertenece el still: director, año, elenco, productora, número referencial del still (generalmente cada kit promocional incluía una serie entre 7 y 30 stills), entre otros. Destacamos que, cuando la fotografía analizada no contaba con pie de información, las anotaciones al dorso (marcas tipo B) servían como respaldo de algunos datos básicos que nos permitían luego consultar bases de datos sobre cine para completar la ficha descriptiva del still. Las anotaciones al dorso fueron escritas a mano por quienes se encargaron en su entonces de organizar el acervo del CU a medida que se incorporaban nuevos ejemplares. Esto nos habla de una cultura cinematográfica propia del cineclubismo y cinefilia a nivel amateur practicada por la comunidad de la institución. Como se verá a continuación, las entrevistas realizadas a actores clave del CU revelan las competencias y conocimientos de su comunidad sobre cinematografía.

\subsection{La memoria y cultura cinematográfica del Cine Universitario a través del relato de su comunidad}

Dentro del diseño metodológico de nuestra investigación estipulamos "Entrevistar actores clave de la comunidad del CU que signifiquen fuentes de información pertinentes a construir conocimiento sobre el género film still, el CU y su comunidad" como uno de los objetivos a cumplir. Esto se tradujo a tres entrevistas realizadas a actores clave dentro de la comunidad del CU con perfiles representativos a las dos dimensiones que diseñamos. Ellos son: (E1) Andrea Armani (miembro de la Comisión Honoraria del CU; archivóloga, actualmente 
reorganizando y mejorando la Biblioteca del CU) ; (E2) Osvaldo Rodríguez (miembro de la comisión honoraria del CU; estuvo a cargo del Archivo del CU, especialmente en el ordenamiento del acervo de film stills durante 40 años) ; (E3) Guillermo Cresci (ex-integrante de la Comisión del CU, uno de los referentes históricos de la institución).

Los tres entrevistados comparten el interés y aprecio por el cine desde distintos roles (profesional de la información (e1) ; coleccionista amateur de cine (e2) y cinéfilo (e3). Las entrevistas fueron individuales, tuvieron un diseño semiestructurado, con el fin de obtener respuestas clave para nuestro estudio (por ejemplo, si los entrevistados conocían el género fotográfico film still), y al mismo tiempo, se dejó un espacio retrospectivo de preguntas abiertas sobre sus experiencias en el CU. La historia oral que nos ofrecieron los entrevistados comprobó nuestro modelo acertado: la importancia que los entrevistados le atribuyen al Archivo y Biblioteca del CU está ligada a la historia social y cultural de la institución como una memoria representativa de su cultura cineclubista. Si bien no conocían al film still por su nombre, sí reconocían su uso en medios de prensa, su adquisición a través de la compra de películas y el kit promocional o press book en el que venían, así como su exhibición en las carteleras de cines en general y en particular en las del CU (véanse ilustraciones 4 y 5). 
Ilustración 4. [Hall del Cine Universitario en la sede de calle Andes 1382 (Año 1970)]

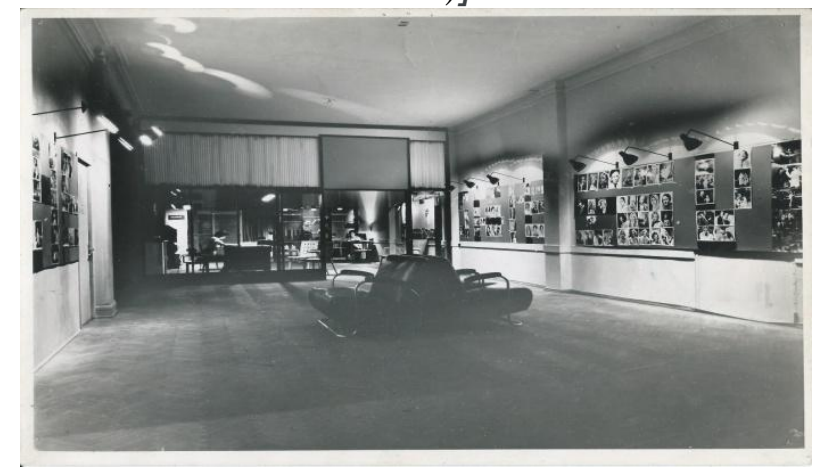

Fuente: Cine Universitario del Uruguay

Ilustración 5. [Film stills expuestas en el hall del Cine Universitario en la sede de

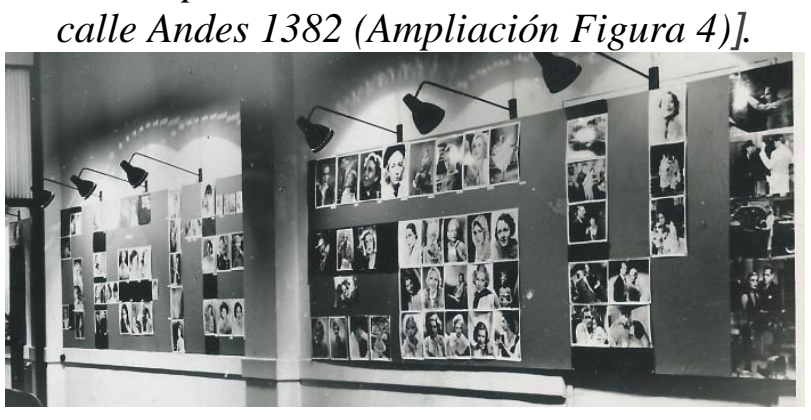

Fuente: Cine Universitario del Uruguay

También hicieron mención a las marcas de uso. En cuanto a las del tipo A, reconocieron el uso de stills en carteleras y vitrinas de cines; en cuanto a las tipo $\mathrm{B}$, destacaron que el acervo actualmente está compuesto por material propio del Archivo del CU, así como por una donación del Cine Club del Uruguay. El trabajo amateur de organización y mantenimiento del acervo estuvo a cargo de Osvaldo Rodríguez (E2) quien se reconoce como coleccionista y cinéfilo desde temprana edad. Fue interesante aprender que las mismas prácticas que llevó a cabo en el acervo las sigue ejercitando para su vida personal (tiene una colección propia de recortes de revistas, fichas técnicas de películas, entre otras). Esto nos habla de un perfil dentro de la comunidad que ha hecho de su cultura cinematográfica una forma de vida.

Los tres entrevistados destacan el valor del cine como una experiencia transformadora para sus vidas y hacen énfasis en el CU como espacio de socialización en torno a intereses comunes así como el potencial que la institución 
tiene en transformarse en una "casa cultural" (E3). En este sentido, reconocemos el valor del film still como catalizador de conversaciones pertinentes al pensar y quehacer cinematográfico.

\subsection{Hacia un modelo de tratamiento documental de fotografía film still: el caso del Cine Universitario}

Existen escasos antecedentes (González López, 2006) sobre el tratamiento documental del film still. Esto nos incita a construir como hemos visto hasta ahora, un marco teórico-práctico plausible de aplicarse al acervo del CU. Paralelamente, las conversaciones en torno al abordaje de la imagen en el paradigma de la información remiten a extensas conversaciones y propuestas desde la archivología y bibliotecología que vuelven compleja la búsqueda de respuestas compatibles con los objetivos del presente proyecto.

Recordamos que nos enfrentamos a una colección audiovisual dentro de un cine no comercial. En consecuencia, la gestión de la misma está naturalmente ligada al perfil y a los procesos socio - históricos de la institución a la que pertenece. Para acercarnos al modelo del tratamiento documental del acervo del $\mathrm{CU}$, entendemos primaria la necesidad de definir las dimensiones que tiene el mismo. En esta instancia, desarrollamos dos.

La primera es interna, y comprende al carácter independiente del acervo, es decir, a las correspondencias histórico-documentales que comparte con el género film still clásico como tal y por tanto, permite enmarcar su análisis y adecuar las herramientas teórico-metodológicas de nuestra disciplina para instrumentar su tratamiento. Por su parte, la segunda dimensión, de tipo externa comprende al contexto en el que encontramos al acervo estudiado, dentro de la institución cineclubista que es el CU y por tanto, involucra a los integrantes de su comunidad. Dado a que el film still fue pensado para exhibirse al público, entonces este último no puede pasar desapercibido en una investigación sobre este género fotográfico. De esta forma, el film still demanda un tratamiento adaptable tanto a su naturaleza visual e inmediata en cuanto a su carácter documental, así como también requiere reconocer "una dimensión cultural del mundo audiovisual, una dimensión que sirve como testimonio de la herencia común de todo el siglo XX" (Caridad, Hernández, Rodríguez \& Pérez, 2011 : 24). Por tanto, las fotografías film stills nos hablan de una herencia cinematográfica que cobra sentido en tanto es 
articulada con el escenario social para el que fue pensada en sus inicios, es decir, su público. Entendemos la presente investigación como una contribución a la preservación y visibilización de esta herencia.

El caso del acervo de film stills del CU supone un punto de partida para hacer uso de los avances tecnológicos tanto en su dimensión interna (preservación, conservación, análisis y manipulación del acervo, entre otros) como también desde su dimensión externa (gestión cultural, rentabilidad, difusión y alfabetización). Es imprescindible contar con la mirada de la comunidad del CU, que singulariza la dimensión social del cineclub como un espacio de intercambio y creación del conocimiento, fenómeno muy cercano al que conocemos desde nuestra disciplina, en el cual las bibliotecas juegan un rol similar como plataforma para que este tipo de relaciones informativas se manifiesten. Esperamos que este proyecto aporte insumos pertinentes a la siguiente discusión.

\section{Discusión}

Primeramente, es necesario detenernos en el género fotográfico film still como tal. Como hemos expuesto, no existe un volumen vasto de literatura publicada sobre la historia de la fotografía film still ni tampoco esta ha gozado de un estudio explícitamente científico incluso durante su época de auge entre 1920 y 1950.

La bibliografía recogida sumada a las instancias de entrevistas realizadas revelan una singular convivencia entre el reconocimiento de la fotografía film still cuando es físicamente visibilizada en contexto por el público (en vitrinas y carteleras por ejemplo) y el descuido - casi absoluto - de la historia y características tanto fotográficas como documentales de este género fotográfico fuera del set de filmación. Es interesante remitir esta desunión a la razón de ser netamente publicitaria del film still en los orígenes de la industria cinematográfica que priorizó su reproducción y distribución masiva con el fin de ingresar en el dominio público de medios y prensa para promover películas. Los stills tendieron a ponderarse más como un medio para un fin que, entre otros aspectos, terminó por eclipsar al complejo oficio y expertise característicos del fotofijas o stillman. En consecuencia, vemos que la falta de reconocimiento de la autoría y la naturaleza descartable de las fotografías aseguraron la subestimación del film still a los ojos de sus públicos mientras simultáneamente calaron en parte de la 
ritualidad del 'ir al cine', del anticipo en el hall o lobby del teatro previa la función, formando así parte imprescindible de la construcción de cultura cinematográfica.

Observamos una paradoja similar entre los términos film still y foto fija. Este último se ha desarrollado en función al primero a la vez que ha adaptado un identidad propia informada por el pensar y quehacer fílmico iberoamericano y este a su vez influenciado por la sensibilidad y filosofía europea primariamente. Sin embargo, la muestra analizada devuelve una marcada presencia de producciones estadounidenses seguidas por las francesas, lo cual nos permite avanzar al siguiente punto de la discusión. En este sentido, debemos aludir a la particularidad del CU como institución cineclubista, la cual responde a una propuesta cultural que dio bienvenida a todo tipo de producciones cinematográficas en cuanto estas respondiesen a un orden mayor abocado a interpelar la realidad y construir conocimiento a través de la crítica del lenguaje fílmico.

A través de la muestra analizada, los datos relevados pueden proyectar un perfil de consumo y gusto cinematográfico en cuanto a los tipos de películas que llegaron al CU y que fueron proyectadas en ese entonces. Así se podría observar, por ejemplo, quiénes eran los actores y las actrices preferidas en su época de apogeo, quiénes se catapultaron más adelante, los directores que sobresalen en esa época, los géneros predominantes, entre otros. En consecuencia, es interesante interpretar la caducidad del formato clásico del film still en papel fotográfico (a diferencia del film still actual que se rige primariamente a través tomas digitalizadas) como la característica única que lo singularizó y lo transformó en un documento visual.

Asimismo, su valor dentro del CU permite reconstruir parte de la memoria institucional a la que pertenece y podría iluminar ciertas problemáticas que rodean la historia del cine uruguayo y la construcción de sus públicos, ciertamente fundamentados en el cineclubismo y cinefilia que supo manifestarse tanto en Uruguay como en la región durante la década de 1950. Es este quizá el espíritu experimental que permitió a los stills del CU conservarse en el tiempo, implícitamente interpretados por sus miembros como objetos necesarios de ser 
coleccionados que solo en un ámbito cineclubista podrían haber perdurado como lo hicieron.

\section{Conclusiones}

La finalidad de esta investigación fue converger la historia del film still con su interpretación documental al sintetizarse a través de un proceso de análisis documental sobre una muestra de 257 fotografías film still pertenecientes a la década de 1950 que componen parte del acervo fotográfico del CU. Los resultados nos devuelven una historia poco documentada en torno al film still y sin embargo clave para la comprensión de la historia del cine en sus inicios. La imagen fotográfica como objeto de información en tiempos actuales supone un tipo de documento masivo que ha cambiado la forma en que las personas se relacionan con la información. Por tanto, nos preguntamos ¿cómo puede el acervo de film stills del CU conjugarse en este presente?

La muestra de film stills estudiada permite acercar a distintas generaciones cineclubistas dentro de la comunidad del CU y traer al presente no solo una memoria y cultura cinematogáfica del pasado sino también ofrecer un mecanismo para su difusión y creación de conocimiento cinematográfico a través de su tratamiento documental y gestión cultural. En tiempos donde la era digital rige nuestras prácticas documentales, debemos hacer uso de sus herramientas y favorecer la conservación, preservación y difusión de este tipo material que puede servir al estudio de la historia del cine en su fotografía, vestuario, la obra de un director, corrientes cinematográficas, entre otros. He aquí la importancia de que sean conservados en las mejores condiciones posibles, siendo la digitalización una solución posible para reconstruir su historia y conservarla a la vez que se sigue construyendo conocimiento en nuevas plataformas y modalidades.

Desde nuestra disciplina debemos reconocer e investigar las transformaciones que han cambiado la manera en que la sociedad accede a y utiliza la misma, siendo la imagen - y por extensión, la imagen en movimiento que supone el cine - un fenómeno de información masivo que ocupa una parte significativa en la vida socio-informativa de las personas. Nuevos tiempos y espacios de innovación y desarrollo profesional se nos presentan en el siglo XXI, y con él nos invita a la reflexión y creatividad que siempre han sido competencias íntegras de nuestra rica 
profesión, siendo hoy más que nunca un presente fuertemente necesitado de ellas. Esperamos desde nuestro trabajo contribuir a su continuo desarrollo.

\section{Referencias bibliográficas}

Archer, F. R., Freyer, E. (1928). Still photography in motion picture work.

Transactions of the Society of Motion Picture Engineering 12 (33), 167172.

Barcelofília. (2014). ALEXIS. Teatre i Cinema. Rambla Catalunya. 90 (19542013). [Fotografía]. Recuperado de:

http://barcelofilia.blogspot.com/2014/05/alexis-teatre-i-cinema-ramblacatalunya.html

Blanchard, W. (1930). Making Stills Tell a Story. American Cinematographer, (5), 12-13.

Boddy, W. (1985). The Studios Move into Prime Time: Hollywood and the Television Industry in the 1950s. Cinema Journal 24 (4), 23-37.

Broitman, A., Eseverri, M. (2018). Renovación y actualidad del cineclubismo en la Ciudad de Buenos Aires. Letra. Imagen. Sonido: Ciudad Mediatizada, (19), 164-180. Recuperado de: https://dialnet.unirioja.es/descarga/articulo/6570877.pdf

Burke, P. (2005). Lo visto y lo no visto: el uso de la imagen como documento histórico. Barcelona: Crítica

Caridad, M., Hernández Pérez, T., Rodríguez Mateos, D., Pérez Lorenzo, B. (2011). Documentación Audiovisual: nuevas tendencias en el entorno digital. Madrid : Síntesis.

Cine Universitario del Uruguay (1970). [Hall del Cine Universitario en la sede de calle Andes 1382 (Año 1970); Figura]. En Resúmenes de actividades, vi. Montevideo: Departamento de Publicaciones del Cine Universitario. 
Cine Universitario del Uruguay (1970). [Film stills expuestas en el hall del Cine Universitario en la sede de calle Andes 1382 (Ampliación Figura 4); Figura]. En Resúmenes de actividades, vi. Montevideo: Departamento de Publicaciones del Cine Universitario.

Costa, J. \& Scavino, C. (2009). Por amor al cine: Historia del Cine Universitario del Uruguay. Montevideo: Ricardo Romero Curbelo.

Flores Huelves, M., Montes Vozmediano, M. (2017). Construyendo cultura visual a través del cartel de cine: análisis de afiches de las sagas cinematográficas. Información, cultura y sociedad, (3), 127-144. Recuperado de: https://dialnet.unirioja.es/descarga/articulo/6560342.pdf

Freund, G. (2017). La fotografía como documento social. Barcelona: GG.

García Torres, A. (2013). La importancia del foto fijas de los años 30 y 40 en la publicidad del cine mexicano: el stillman un oficio olvidado. (Monografía de grado, Universidad Nacional Autónoma de México, Ciudad Universitaria).

Gomery, D. (2004). The Images in Our Minds.: Film Stills and Cinema History. The Princeton University Chronicle, 65 (3), 502-520.

González López, P. (2006). Análisis, descripción, catalogación y conservación de fondos documentales de foto fija para cine. Una experiencia de colaboración entre el Departamento de $H^{a}$ del Arte de la Universidad de Barcelona y la filmoteca de Cataluña. En XIV Congreso nacional de Historia del Arte. Trabajo presentado en el congreso de la Universidad de Málaga, Málaga.

Jacobs, S. (2010). The History and Aesthetics of the Classical Film Still. History of Photography 34(4), 373-386.

Kemmis, S. \& McTaggart, R. (1988). The Action research planner (3rd ed). Deakin University. 
MacKenzie, D. (1934). The life of a Stillman. The International Photographer, $5(12), 22$.

Meza Castro, M. (2018). El recurso de información y comunicación visual: imagen. Apuntes en torno a las Ciencias de la Información y Bibliotecología. E-Ciencias De La Información,8(2). https://doi.org/10.15517/eci.v8i2.29956

Solórzano-Ariza, A., Toro-Tamayo, L. C., \& Vallejo-Echavarría, J. C. (2017). Memoria fotográfica: la imagen como recuerdo y documento histórico. Revista Interamericana de Bibliotecología, 40(1), 73-84. Doi: 10.17533/udea.rib.v40n1a0

Sony Entertaiment (2019) Proud: The 28-year-old Oscar-nominated actress puts on an impressive performance as the relatively bubbly Tate who goes to the movies to watch herself in 1968 Dean Martin film The Wrecking Crew. [Figura]. Recuperado de:

https://www.dailymail.co.uk/tvshowbiz/article-7054657/Once-TimeHollywood-trailer-Brad-Pitt-Leonardo-DiCaprio-Luke-Perry-final$\underline{\text { role.html }}$

Tuñón, J. (2002, marzo 4). Entre fotos te veas: del cine al still. [Publicación de blog]. Recuperado de: https://centrodelaimagen.files.wordpress.com/2010/03/texto-entre-fotoste-veas julia-tunon.pdf

Universal Pictures Company Inc. (1955). [Still de la película Tarántula (1955) de Jack Arnold] [Fotografía]

Virué Escalera, L. (2012). La fotografía fija en los Abrazos rotos: categorías y uso en la construcción de la marca Almodóvar. Fotocinema , (5), 112-145. Recuperado de: https://dialnet.unirioja.es/descarga/articulo/4147468.pdf 


\section{Notas}

${ }^{1}$ Espacio de Formación Integral (EFI) "Investigación-Acción aplicada a proyectos estudiantiles de extensión"; el trabajo fue titulado "Film Stills: Documentación Audiovisual y el caso del Cine Universitario", y realizado en el marco de la Unidad Curricular Documentación Audiovisual de la Licenciatura en Bibliotecología (FICUDELAR). La tutoría estuvo a cargo de la docente Mag. Djamila Romani (Instituto de Información - FIC) durante los meses agosto - noviembre del 2017.

${ }^{2}$ Proyecto de Extensión Universitaria "Film Stills: hacia la recuperación del acervo fotográfico del Cine Universitario", presentado al llamado de la Unidad Proyectos Estudiantiles de Extensión Universitaria 2018. Seleccionado y financiado por la Unidad de Apoyo a la Extensión y Actividades en el Medio (UDEA - FIC) y se ejecutó durante los meses agosto - noviembre del 2018. Contó con la tutoría de la docente Mag. Djamila Romani (Instituto de Información - FIC) y el apoyo del referente y representante de la Udelar en la Comisión Honoraria del Cine Universitario, docente Luis Dufuur (Instituto de Comunicación - FIC). Asimismo, en esa instancia, se logró incorporar al equipo al estudiante de la Licenciatura en Comunicación Nicolás Erramuspe, también miembro de la Comisión Honoraria del Cine Universitario.

3 El proyecto PAIE "Film Stills: la fotografía como memoria visual del Cine Universitario" se ejecutó entre los meses abril-octubre del 2019. Contó con la tutoría de la docente Mag. Djamila Romani (Instituto de Información - FIC). 